\title{
Onsager's reciprocal relations for electroacoustic and sedimentation: Application to (concentrated) colloidal suspensions
}

\author{
S. Gourdin-Bertin ${ }^{1,2}$ and C. Chassagne $e^{1,3}$ \\ ${ }^{1}$ Sorbonne Universités, UPMC Université Paris 06, UMR 8234, PHENIX, F-75005 Paris, France \\ ${ }^{2}$ CNRS, UMR 8234, PHENIX, F-75005 Paris, France \\ ${ }^{3}$ Department of Environmental Fluid Mechanics, TU Delft, Stevinweg 1, 2628 CN Delft, The Netherlands
}

(Received 31 March 2015; accepted 8 May 2015; published online 20 May 2015)

\begin{abstract}
In this article, the relations for electroacoustic phenomena, such as sedimentation potential, sedimentation intensity, colloid vibration potential, colloid vibration intensity/current, or electric sonic amplitude, are given, on the basis of irreversible thermodynamics. This formalism allows in particular to discuss the different expressions for concentrated suspensions found by various authors, which are of great practical interest. It was found that some existing expressions have to be corrected. Relations between the electrophoretic mobilities assessed by the different experiments are derived. (C) 2015 AIP Publishing LLC. [http://dx.doi.org/10.1063/1.4921375]
\end{abstract}

\section{INTRODUCTION}

Electroacoustic phenomena, such as Colloid Vibration Potential (CVP), Colloid Vibration Intensity/Current (CVI), or Electric Sonic Amplitude (ESA), allow to assess the properties of (concentrated) suspensions and in particular the zeta potential of individual particles. The commercially available devices measuring the CVP and ESA phenomena are routinely used by research groups and industry. Since the initial experiments of Rutgers on CVP in $1946,{ }^{1}$ experimental devices to measure the CVP response of colloidal suspensions have been commercialized by Marlow ${ }^{2}$ in 1988, by O'Brien ${ }^{3}$ for ESA the same year, and a few years later for CVP by Dukhin. ${ }^{4}$ These devices come with different theories to interpret the measured response. Theories for the electroacoustics response of colloids were developed separately, first by Henry and Booth, Enderby in $1952,{ }^{5}$ by O'Brien and coworkers from the 1980 s onwards, ${ }^{3-6}$ and by Ohshima and coworkers ${ }^{7,8}$ and by Dukhin and coworkers ${ }^{9}$ in the same decades.

On the other hand, even though the effect was discovered by Dorn in 1880, Sedimentation Potential (SP) experiments are scarce. ${ }^{11,12}$ Theories for interpreting the sedimentation potential data have been formulated by Booth in 1954, for low zeta potentials but all particle sizes and double layer thicknesses. ${ }^{13}$ In 1984, Ohshima, Healy, White, and O'Brien ${ }^{7}$ derived the sedimentation velocity of a single charged sphere and the sedimentation potential of a dilute suspension, for all zeta potentials and all particle sizes and double layer thicknesses.

All the previously cited theories were derived making use of the "electrokinetic set of equations," which include Poisson, balance equation for ions, and Navier-Stokes. Another approach is possible, based on irreversible thermodynamic considerations. The corresponding relations between fluxes and gradients and the famous "reciprocal relations" were introduced by Onsager in 1931. In 1952, de Groot, Mazur, and Overbeek ${ }^{14}$ derived the Onsager reciprocal relations for sedimentation. In 2014, Chassagne and Bedeaux ${ }^{15}$ extended the results of de Groot, Mazur, and Overbeek to the electroacoustic phenomena. Ohshima et $a .^{7}$ demonstrated that the Onsager relations were respected in the case of the sedimentation velocity and potential theory presented in their paper, for a dilute suspension of charged spherical colloidal particles. Ohshima ${ }^{8}$ later made an analogy between SP and CVP to establish Onsager relationships in this case. Dukhin et al. used the Onsager relation for electroosmosis/streaming potential to derive an expression for CVI. ${ }^{9,10}$

Our first goal, in this paper, is to check whether the theories found by different authors, using different assumptions, are in agreement with the Onsager relations. Our second goal is to present the link between the different electrophoretic mobilities assessed by different theories/experiments. This last point has been a matter of debate since 15 years. ${ }^{6,9,10}$ We hope that the present article will clarify this issue.

In Sec. II, we briefly recall important results regarding the Onsager relations for sedimentation and electroacoustics. These relations were derived in Refs. 14-16 by writing the entropy production from which the linear force-flux relations follow. Using the fluxes-gradients approach then enables us, in Sec. III, to comment on the theories presented by different authors. We will discuss general results regarding the volumefraction dependence, which are of high practical importance. In the conclusion, we present the general relation between the electrophoretic mobilities found by electrophoretic mobilities measurements, from theoretical/numerical considerations, and $\mathrm{SP}(\mathrm{SI}) / \mathrm{CVP}(\mathrm{CVI}) / \mathrm{ESA}$ measurements.

\section{THEORY}

In this section, we give the entropy production derived for sedimentation and electroacoustics. By writing the entropy production, the forces and the fluxes needed to setup the forces-fluxes relations can be correctly defined. In particular, the forces and fluxes thus defined have the dimensions that ensure that the cross coefficients (defined below) have the same dimensionality. This also enables to have a coherent definition for the signs. The general derivations of the equations given in this section can be found in Refs. 14-16. As is discussed 
in more detail in Ref. 16, and as was hinted by Ohshima in Ref. 8, the relations for sedimentation can be seen as the lowfrequency limit of the electroacoustic equations.

\section{A. Sedimentation}

The total entropy production $\sigma$ in the case of sedimentation was found to be

$$
\sigma=\frac{\mathbf{I} \cdot \mathbf{E}}{T}+\frac{\mathbf{g} \cdot \mathbf{J}^{l a b}}{T} .
$$

The corresponding force-flux relations derived from the entropy production were found to be

$$
\begin{aligned}
\mathbf{J}^{l a b} & =m_{P P} \mathbf{g}+m_{P E} \mathbf{E}, \\
\mathbf{I} & =m_{E P} \mathbf{g}+m_{E E} \mathbf{E},
\end{aligned}
$$

where $\mathbf{J}^{l a b}$ represents the total mass flow in the reference frame of the laboratory (superscript $l a b$ ) and $\mathbf{J}^{l a b}=\rho \mathbf{v}$, where $\rho$ is the density of the suspension and $\mathbf{v}=\mathbf{v}_{b a r}^{\text {lab }}$ the velocity of the center of mass of the system in the reference frame of the laboratory (from now on, the subscript lab and superscript bar will be dropped). The electric current is defined by the symbol $\mathbf{I}$, the electric field by $\mathbf{E}$, and the gravitational field by $\mathbf{g}$. The coefficients of proportionality $m_{i j}$ can either be measured or estimated from theories, and Onsager's relation gives that the cross coefficients respect the relation $m_{P E}=m_{E P}$.

For colloids, assuming that the total mass of the colloidal particles is much larger than the total mass of the ions, it was found that in good approximation ${ }^{15}$

$$
\begin{gathered}
\rho=\phi \bar{\rho}_{p}+(1-\phi) \bar{\rho}_{w}, \\
\rho \mathbf{v}=\phi \bar{\rho}_{p} \mathbf{v}_{p}+(1-\phi) \bar{\rho}_{w} \mathbf{v}_{w},
\end{gathered}
$$

where $\phi$ is the volume fraction of the colloidal particles, and $\bar{\rho}_{p}$ and $\bar{\rho}_{w}$ are the absolute densities of the colloidal particles and the solvent (water). The bars on the densities are introduced to avoid confusion with the definition of other densities introduced in Ref. 14 and subsequently used in Refs. 15 and 16. The velocities $\mathbf{v}_{p}$ and $\mathbf{v}_{w}$ represent the velocities of the colloidal particles and the solvent in the reference frame of the laboratory.

Using the fact that the total volume flux is zero in the case of sedimentation, it is possible to write ${ }^{15}$

$$
\phi \mathbf{v}_{p}+(1-\phi) \mathbf{v}_{w}=0
$$

From which one gets

$$
\mathbf{v}=\frac{\bar{\rho}_{p}-\bar{\rho}_{w}}{\rho} \phi \mathbf{v}_{p}=\frac{\Delta \rho}{\rho} \phi \mathbf{v}_{p} .
$$

The entropy production can now be rewritten in the form

$$
\sigma=\frac{\mathbf{I} \cdot \mathbf{E}}{T}+\frac{\Delta \rho}{\rho} \phi \frac{\boldsymbol{\nabla} P \cdot \mathbf{v}_{p}}{T} .
$$

From the entropy production, new flux-force relations can be derived,

$$
\begin{aligned}
\mathbf{v}_{p} & =l_{P P} \Delta \rho \phi \mathbf{g}+l_{P E} \mathbf{E}, \\
\mathbf{I} & =l_{E P} \Delta \rho \phi \mathbf{g}+l_{E E} \mathbf{E} .
\end{aligned}
$$

Note that since the system is at mechanical equilibrium, a pressure gradient is generated by the gravitational field $(\nabla P$ $=\rho \mathbf{g}) \cdot{ }^{16}$ The reciprocal Onsager relation to be verified is $l_{P E}$ $=l_{E P}$ and it follows that the sedimentation intensity (SI) can be defined as

$$
\mathrm{SI}=(\mathbf{I})_{E=0}=\Delta \rho \phi\left(\frac{\mathbf{v}_{p}}{\mathbf{E}}\right)_{g=0} \mathbf{g} .
$$

The term $l_{P E}=\left(\mathbf{v}_{p} / \mathbf{E}\right)_{g=0}$ is by definition the electrophoretic mobility of the particle measured at zero total volume flux condition (in the laboratory frame of reference), i.e., $\mu_{\mathrm{E}}^{\text {lab, vol. flux }=0}$, and can be identified with $\mu_{\mathrm{E}}$, i.e., the electrophoretic mobility obtained from standard electrophoretic mobility measurements (see Appendix A). The SP of the suspension is obtained from the last line of Eq. (7),

$-\nabla(\mathrm{SP})=(\mathbf{E})_{I=0}=\frac{-\mu_{\mathrm{E}}}{K} \Delta \rho \phi \mathbf{g}=\frac{-\mu_{\mathrm{E}}}{K} \Delta \rho \phi \frac{\boldsymbol{\nabla} P}{\rho}$.

Note that of course $\mathbf{g}=(\mathbf{g})_{I=0}=(\mathbf{g})_{E=0}$. In setting-up the last equation, we have used the hydrostatic equation: $\nabla P=\rho \mathrm{g}$.

\section{B. CVP/CVI}

The total entropy production $\sigma$ in the case of electroacoustics was found to be

$$
\sigma=\frac{\mathbf{I} \cdot \mathbf{E}}{T}+\frac{1}{T} \frac{\boldsymbol{\nabla} P}{\rho} \cdot \mathbf{J}^{v o l} .
$$

The corresponding forces-fluxes relations derived from the entropy production were found to be

$$
\begin{aligned}
\mathbf{J}^{v o l} & =b_{P P} \frac{\boldsymbol{\nabla} P}{\rho}+b_{P E} \mathbf{E}, \\
\mathbf{I} & =b_{E P} \frac{\boldsymbol{\nabla} P}{\rho}+b_{E E} \mathbf{E},
\end{aligned}
$$

where $\mathbf{J}^{v o l}$ represents the total mass flow in the reference frame of the total volume flow (superscript vol). The coefficients of proportionality $b_{i j}$ can either be measured or estimated from theories, and the Onsager relation to be satisfied is $b_{P E}=b_{E P}$. Note that because of electroneutrality, the electric current is independent of the frame of Ref. 15. Along the lines given in Subsection II A and in Ref. 15, and using the fact that the total volume flux is evidently zero in the reference frame of the total volume flux, one can modify the entropy production into

$$
\sigma=\frac{\mathbf{I} \cdot \mathbf{E}}{T}+\frac{1}{T} \frac{\Delta \rho}{\rho} \phi \boldsymbol{\nabla} P \cdot \mathbf{v}_{p}^{v o l} .
$$

The new flux-force relation in this case can be written as

$$
\begin{aligned}
\mathbf{v}_{p}^{v o l} & =d_{P P} \Delta \rho \phi \frac{\boldsymbol{\nabla} P}{\rho}+d_{P E} \mathbf{E}, \\
\mathbf{I} & =d_{E P} \Delta \rho \phi \frac{\boldsymbol{\nabla} P}{\rho}+d_{E E} \mathbf{E} .
\end{aligned}
$$

The relation between $\mathbf{v}_{p}^{l a b}$ and $\mathbf{v}_{p}^{v o l}$ is given by

$$
\mathbf{v}_{p}^{l a b}=\mathbf{v}_{p}^{v o l}+\mathbf{v}_{v o l}^{l a b}
$$


The total volume flux in the reference frame of the laboratory $\mathbf{v}_{v o l}^{l a b}$ is created by the applied pressure gradient generating the acoustic wave and ensures that the liquid compresses and dilates locally. An estimation of $v_{v o l}^{l a b}$ for the liquid can be obtained by considering the value for the acoustic impedance $z$ for water and the typical amplitude $P$ of the applied pressure gradient which gives

$$
v_{v o l}^{l a b} \simeq \frac{P}{z} \simeq \frac{75000}{1.5 \times 10^{6}}=5 \times 10^{-2} \mathrm{~m} / \mathrm{s} .
$$

An estimation for the electrophoretic velocity of a colloidal particle at zero gravitational field is given by

$$
\begin{aligned}
\left(v_{p}^{l a b}\right)_{g=0} & \simeq \frac{\varepsilon_{0} \varepsilon_{1}}{\eta} \zeta E \\
& \simeq \frac{8.85 \times 10^{-12} \times 80}{10^{-3}} 100 \times 10^{-3} \frac{100}{10 \times 10^{-2}} \\
& \simeq 7 \times 10^{-5} \mathrm{~m} / \mathrm{s},
\end{aligned}
$$

where $\varepsilon_{0} \varepsilon_{1}$ is the dielectric permittivity of water and $E$ the electric field is taken to be the typical electric field in electrophoretic measurements, i.e., $10 \mathrm{~V} / \mathrm{cm}$. It is therefore clear that the electrophoretic velocity of a colloidal particle cannot be measured directly from electroacoustic measurements and that in good approximation $\mathbf{v}_{p}^{v o l} \simeq-\mathbf{v}_{v o l}^{l a b}$. The electrophoretic mobility can however be obtained making use of the Onsager relation $d_{P E}=d_{E P}$,

$$
(\mathbf{I})_{E=0}=\left(\frac{\mathbf{v}_{p}^{l a b}-\mathbf{v}_{v o l}^{l a b}}{\mathbf{E}}\right)_{\nabla P=0} \frac{\Delta \rho}{\rho} \phi(\nabla P)_{E=0} .
$$

The velocity $\mathbf{v}_{p}^{v o l}=\left(\mathbf{v}_{p}^{l a b}-\mathbf{v}_{v o l}^{l a b}\right)$ can be related to the electrophoretic velocity measured during standard electrophoretic mobility measurements, for which no acoustic wave is applied and therefore $\mathbf{v}_{v o l}^{l a b}=0$. The term $\left(\left(\mathbf{v}_{p}^{l a b}-\mathbf{v}_{v o l}^{l a b}\right) / \mathbf{E}\right)_{\nabla P=0}$ is then by definition the electrophoretic mobility of the particle measured at zero total volume flux condition (in the laboratory frame of reference), i.e., $\mu_{\mathrm{E}}^{\text {lab, vol. flux }=0}$, and can be identified with $\mu_{\mathrm{E}}$, i.e., the electrophoretic mobility obtained from standard electrophoretic mobility measurements. For more details, we refer to Appendix A, where expressions for the electrophoretic mobility are derived and discussed for the cases encountered in theoretical derivations, in CVI/CVP, and in ESA. We therefore get in the case of CVI/CVP that $\left(\mathbf{v}_{p}^{l a b}-\mathbf{v}_{v o l}^{l a b}\right)=\mu_{\mathrm{E}} \mathbf{E}$ and the CVI is given by

$$
\mathrm{CVI}=(\mathbf{I})_{E=0}=\mu_{\mathrm{E}} \frac{\Delta \rho}{\rho} \phi(\nabla P)_{E=0} .
$$

This result may be expressed using the different expressions for electrophoretic mobility found in Appendix A. Using the electrophoretic mobility relative to the solvent $\mu_{0}$, which is generally the one found from theoretical derivations, we find

$$
\mathrm{CVI}=(\mathbf{I})_{E=0}=\mu_{0} \frac{\bar{\rho}_{p}-\rho}{\rho} \phi(\nabla P)_{E=0},
$$

where we used the fact that $\bar{\rho}_{p}-\rho=\Delta \rho(1-\phi)$. Indeed, as the colloid moves forward, there is a forward volume flux; the condition for the Onsager relation is that there is no such volume flux, so the whole suspension undergoes the backflow; therefore, the density associated with the backflow is $\rho$. With the electrophoretic mobility relative to the center-of-mass,
$\mu_{\mathrm{E}}^{\text {lab, mass. flux }=0}=\mu_{\mathrm{E}}^{*}$, which is used by O'Brien in Ref. 6, by some types of numerical simulations, ${ }^{17}$ and discussed more in detail in Subsection III C, we find

$$
\mathrm{CVI}=(\mathbf{I})_{E=0}=\mu_{\mathrm{E}}^{*} \frac{\bar{\rho}_{p}-\bar{\rho}_{w}}{\bar{\rho}_{w}} \phi(\boldsymbol{\nabla} P)_{E=0} .
$$

From the last line of Eq. (13), we also get the colloid vibration field and CVP,

$$
\begin{aligned}
(\mathbf{E})_{I=0} & =\frac{-\mu_{\mathrm{E}}}{K} \frac{\Delta \rho}{\rho} \phi(\nabla P)_{I=0}, \\
\mathrm{CVP} & =\frac{\mu_{\mathrm{E}}}{K} \frac{\Delta \rho}{\rho} \phi \Delta P,
\end{aligned}
$$

where $\Delta P=\left(P_{2}-P_{1}\right)$ is the pressure difference between the two electrodes, located at any odd multiple of half the wavelength (see Fig. 2). $d_{E E}$ can be identified with $K$ the electric conductivity of the suspension. Equations (17) and (20) represent, respectively, the CVI and vibration electric field (linked to the CVP through the distance between electrodes) of the suspension.

This formula may be rewritten with a mass fraction. The mass fraction is by definition $\phi_{w t}=\phi \rho_{p} / \rho$ and our formula becomes

$$
(\mathbf{E})_{I=0}=\frac{-\mu_{\mathrm{E}}}{K} \frac{\Delta \rho}{\rho_{p}} \phi_{w t}(\nabla P)_{I=0}
$$

which is an useful result for experimentalists. This relation is valid for any mass fraction. The electrophoretic mobility $\mu_{\mathrm{E}}$ is a function, generally complicated, of the mass (or volume) fraction of the colloidal particles and is still taken under a zerovolume-flux condition.

\section{DISCUSSION}

The relations found in Sec. II will now be compared to the result obtained from a simple test case (Subsection III A 1) by solving the relevant electrokinetic set of equations. The reciprocal relations found for SP, CVP, and CVI by various authors in Refs. 8, 20, 18, 10, and 9 are then commented. For low volume fractions, the relations found for SP by Ohshima et al. $^{7}$ and by Enderby ${ }^{5}$ for CVP by solving the set of electrokinetic/electroacoustic relations do respect the Onsager reciprocal relations. We show that the reciprocal expressions found for $\mathrm{SP}^{8}$ and $\mathrm{CVP}^{20}$ for high volume fractions have to be corrected. The expressions for $\mathrm{CVI}^{10,9}$ and $\mathrm{ESA}^{3-6}$ are then discussed for both dilute and concentrated suspensions.

\section{A. Sedimentation}

\section{Sedimentation intensity}

In this subsection, we will test whether the Onsager relationship, Eq. (8), is respected for a simple test case. The relation to be satisfied, Eq. (8), reads

$$
(\mathbf{I})_{E=0}=\Delta \rho \phi \mu_{\mathrm{E}} \mathbf{g} \text {. }
$$

We write Newton's equation of motion for a colloidal particle immersed in an electrolyte solution in a suspension of volume 
fraction $\phi$,

$$
-6 \pi \eta a\left(\mathbf{v}_{p}-\mathbf{v}_{w}\right)+m_{p}\left(1-\rho / \bar{\rho}_{p}\right) \mathbf{g}+q \mathbf{E}=\mathbf{0},
$$

where we assumed the colloidal particle to have an electric charge $q$, to be a sphere of radius $a$ in a solvent of viscosity $\eta$, and have a friction coefficient $6 \pi \eta a$. The density $\rho$ is given in Eq. (3). From Eqs. (3) and (4),

$$
\begin{aligned}
1-\frac{\rho}{\bar{\rho}_{p}} & =(1-\phi) \frac{\Delta \rho}{\bar{\rho}_{p}}, \\
\mathbf{v}_{p}-\mathbf{v}_{w} & =\frac{1}{1-\phi} \mathbf{v}_{p} .
\end{aligned}
$$

Using these two equations and Eq. (24), we get an expression for $\mathbf{v}_{p}$,

$$
\mathbf{v}_{p}=(1-\phi)^{2} \frac{m_{p}}{6 \pi \eta a} \frac{\Delta \rho}{\bar{\rho}_{p}} \mathbf{g}+\frac{q_{p}}{6 \pi \eta a}(1-\phi) \mathbf{E} .
$$

We can identify

$$
\mu_{\mathrm{E}}=\left(\frac{\mathbf{v}_{p}}{\mathbf{E}}\right)_{g=0}=\frac{q_{p}}{6 \pi \eta a}(1-\phi)=\mu_{0}(1-\phi)
$$

as the electrophoretic mobility of a colloid, where the electric field arises from the settling of the (charged) colloids under gravitational field. The mobility $\mu_{0}$ is defined in Appendix A and represents the theoretical mobility of a colloidal particle (in the laboratory frame of reference) defined with no particular conditions for the volume or mass fluxes. In case $\phi \ll 1$, we recover the result obtained by Hückel for a sphere with no polarizable double layer moving in an electric field, without the influence of its neighbours. More elaborate models exist, which take into account the polarization of the colloid's double layer, for estimating $\mu_{\mathrm{E}}$. In order to setup these models, Eq. (24) should be modified so as to account for the spatial variation of $E$ in the neighborhood of the colloid. In the case considered here, since $E$ is assumed constant everywhere, we are in the situation where the double layer around the particle is much larger than the particle size (i.e., $\kappa a \ll 1$, where $\kappa$ is the inverse of the Debye length and $a$ the radius of the colloidal particle). For $\phi \simeq 1$, we get $\mu_{\mathrm{E}} \simeq 0$, which immediately derives from the fact that the total volume flow is zero, see Eq. (4), as the packing of spheres then prevents any volume flow.

In the case of hindered settling, various expressions have been developed for spherical colloids to account for the change in settling velocity of a particle; as compared to the case, this particle is free to settle. After rearranging Eq. (26), and using the definition

$$
\bar{m}_{p}=m_{p}\left(1-\bar{\rho}_{w} / \bar{\rho}_{p}\right)=m_{p} \Delta \rho / \bar{\rho}_{p}
$$

the settling of a particle in the absence of electric field is found to be

$$
\mathbf{v}_{\text {sedim }}=\left(\mathbf{v}_{p}\right)_{E=0}=\frac{\bar{m}_{p}}{6 \pi \eta a}\left(1-\phi \frac{\Delta \rho}{\bar{\rho}_{p}}\right)(1-\phi) \mathbf{g} .
$$

The expression for the modified settling velocity, in case of hindered settling, is generally given by

$$
\mathbf{v}_{\text {sedim }}^{\text {hindered settling }}=\frac{\bar{m}_{p}}{6 \pi \eta a} f(\phi),
$$

where $f(\phi)=1$ when $\phi \ll 1$ and decreases when $\phi$ becomes larger. For $\phi=1, \mathbf{v}_{\text {sedim }}=0$. A widely used empirical relation for $f(\phi)$ due to Richardson and $\mathrm{Zaki}^{19}$ gives

$$
\mathbf{v}_{\text {sedim }}^{\text {hindered settling }}=\frac{\bar{m}_{p}}{6 \pi \eta a}(1-\phi)^{n},
$$

where $n$ is an empirically determined exponent usually between 2.5 and 5. The difference between Eqs. (29) and (31) arise from the fact that in Eq. (29), the volume fraction dependence comes solely from setting the total volume flow equal to zero, whereas in Eq. (31), interparticular interactions and hydrodynamical effects are also considered.

The electric current is defined by

$$
\begin{aligned}
\mathbf{I} & =\sum_{k=1}^{n} q_{k} n_{k} \mathbf{v}_{k} \\
& =q_{p} n_{p} \mathbf{v}_{p}+\sum_{k=2}^{n-1} q_{k} n_{k} \mathbf{v}_{k},
\end{aligned}
$$

where $q_{k}$ is the charge of particle $k$ (ion or colloid) and $n_{k}$ the number of particles $k$ per unit volume. For simplicity, we have taken $k=1$ to represent the colloid. Let us assume that the electrolyte ions only and mainly contribute to the electric conductivity $K$. This implies in particular that the ionic velocities do only depend on $\mathbf{E}$ and not on $\mathbf{g}$. The colloid contributes to the current with a factor equal to $q_{p} n_{p} \mathbf{v}_{p}$. We assume that the colloids' counterions move with a velocity equal to the one of water, which is a good assumption in the case studied here as $\kappa a \ll 1$. This implies that the counterions contribute to the current by a factor $-q_{p} n_{p} \mathbf{v}_{w}$. We obtain

$$
\mathbf{I}=\simeq q_{p} n_{p}\left(\mathbf{v}_{p}-\mathbf{v}_{w}\right)+K \mathbf{E} .
$$

Using Eqs. (24) and (25) for estimating $\left(\mathbf{v}_{p}-\mathbf{v}_{w}\right)$, we get

$$
(\mathbf{I})_{E=0}=\phi \frac{q_{p}}{6 \pi \eta a}(1-\phi) \Delta \rho \mathbf{g} .
$$

We find that

$$
l_{P E}=\mu_{\mathrm{E}}=\frac{q_{p}}{6 \pi \eta a}(1-\phi)=l_{E P}
$$

and the Onsager relation holds for any volume fraction, provided that $\kappa a$ is low.

\section{Sedimentation potential}

Ohshima et al. ${ }^{7}$ checked whether the Onsager relation was respected, for any $\kappa a$ and zeta potentials, in the case of low volume fractions, using Eq. (8),

$$
(\mathbf{E})_{I=0}=\frac{-\mu_{\mathrm{E}}}{K} \Delta \rho \phi \mathbf{g} \text {. }
$$

In principle, Eq. (36) can be used also for high volume fractions. The restriction is that the system investigated remains isotropic.

For a suspension of colloidal dielectric particles of any volume fraction $\phi$, it can be demonstrated that (see Appendix B or p. 279 in Ref. 10)

$$
K=K_{1} \frac{1+2 \phi \beta}{1-\phi \beta},
$$

where $K_{1}$ is the ionic conductivity (the conductivity of the solution, i.e., without the colloidal particles) and $\beta$ the dipole 
coefficient for a colloidal particle, which could, in principle, depend on the interaction with nearest neighbours. We have dropped the twiddles on the symbols for conductivity for convenience, but in the general case, the conductivities would be complex and frequency-dependent. We get

$$
\mathbf{E}_{I=0}=\frac{-\Delta \rho \phi \mu_{\mathrm{E}}(1-\phi \beta)}{K_{1}(1+2 \phi \beta)} \mathbf{g} .
$$

For small zeta potentials, $\beta \simeq-1 / 2$ and

$$
\mathbf{E}_{I=0}=\frac{-\Delta \rho \phi \mu_{\mathrm{E}}(1+\phi / 2)}{K_{1}(1-\phi)} \mathbf{g} .
$$

This expression should be compared with Eq. (8.8) of Ohshima in Ref. 8, who gives a relation for small zeta potentials. We note that the term $(1+\phi / 2) /(1-\phi)$ is inverted in his equation compared to ours. (There is also a sign difference, but this is simply due to a typographical error, as the reader can see by considering Eqs. (8.6) and (8.7) in that same paper.) The equations equivalent to Eq. (7) derived by Ohshima are similar to ours. The electric current and electric field he defines are, as in our case, the average current and field (i.e., averaged over a representative volume element), but noted, in his case, $\langle i\rangle$ and $\langle E\rangle$. The relation equivalent to our Eq. (8) reads, in Ohshima's case (see Eq. (8.4) in Ref. 8),

$$
\left(\frac{\mathbf{v}_{p}}{\langle\mathbf{E}\rangle}\right)_{g=0}=\frac{1}{\Delta \rho \phi}\left(\frac{\langle\mathbf{i}\rangle}{\mathbf{g}}\right)_{\langle E\rangle=0} \cdot
$$

The symbol $E$ is used by Ohshima to define the "applied electric field in the absence of gravitational field" as stated in Ref. 8. This electric field is defined at the boundary of the cell (at $r=b$ ) as can be seen in Eq. (2.28) in that article. This electric field is therefore not the macroscopic electric field $\langle E\rangle$ as defined in Eq. (4.1) in Ohshima's article. This is schematized in Fig. 1.

The best way to understand the relation between Ohshima's $E$ and $\langle E\rangle$ is to consider the Eq. (4.4) in Ref. 8, which gives the average electric current (in the absence of the gravitational field, i.e., as is done in electrophoresis and conductivity measurements),

$$
\langle i\rangle=K\langle E\rangle=K_{1} E .
$$

By comparing Eq. (41) to Eq. (37), we deduce that

$$
\langle E\rangle=\frac{1-\phi}{1+\phi / 2} E
$$

which is indeed Eq. (A4) derived by Ohshima in Ref. 8 for $\beta \simeq-1 / 2$. Instead of looking at Eq. (41) in the context of the cell model described by Ohshima, we will let it describe the situation where the electric current $\langle i\rangle$ is taken to be the same in two experiments: in the first experiment, the system considered is an electrolyte suspension of conductivity $K_{1}$ where the application of a current $\langle i\rangle$ gives rise to an electric field defined by $E=\langle i\rangle / K_{1}$, and in the second experiment, the system considered is made of colloidal particles suspended in the same electrolyte solution of conductivity $K_{1}$ where the application of a current $\langle i\rangle$ gives rise to an electric field defined by $\langle E\rangle=\langle i\rangle / K$. In other words, in our case, the electric field called $E$ by Ohshima should be named "the average electric field in an electrolyte solution of conductivity $K_{1}$ in the absence of colloidal particles when an electric current $\langle i\rangle$ is applied in the absence of gravitational field." As implied by Eq. (41), it would also be more correct in our case to call $\langle i\rangle$ the "applied electric current" since it is taken to be the same in both experiments (the electrolyte solution without colloidal particles (experiment 1 ) and with colloidal particles (experiment 2)).

Ohshima defines (Eq. (3.1) in Ref. 8) the electrophoretic velocity of his colloidal particle to be

$$
\mathbf{v}_{p}=\mu_{\mathrm{E}} \mathbf{E} \text { (Ohshima), }
$$

where $E$ is again the "average electric field in an electrolyte solution of conductivity $K_{1}$ in the absence of colloidal particles, when a current $\langle i\rangle$ is applied, in the absence of the gravitational field." Note that this is equivalent to the electric field defined by Ohshima, which is the electric field at the boundary of the cell in which each colloidal particle is embedded. The fact that Ohshima calls it "the applied electric field" is probably
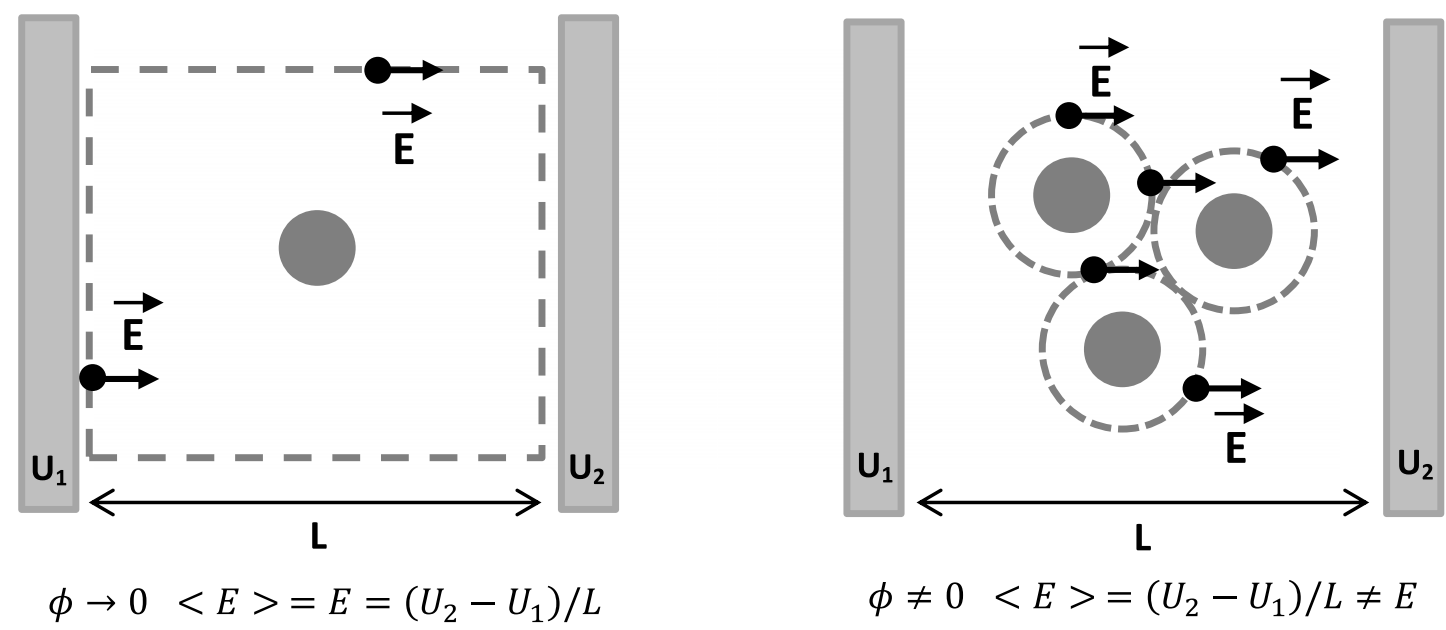

FIG. 1. Definitions of the "applied electric field" E and the "macroscopic electric field" $\langle$ E $\rangle$ as defined by Ohshima in Ref. 8. Left: case of low volume fraction. The dashed region represents the region far from the particle where the electric field is equal to the applied one. Right: case of high volume fraction. The dashed circles of radius $b$ represent the boundary of the cell for each particle; the volume fraction of the colloid is defined by $\phi=(a / b)^{3}$, where $a$ is the radius of the colloidal particle. At this boundary, the electric field $\mathrm{E}$ is similar to the electric field E defined on the left figure. 
because he makes the analogy with the dilute suspension case, where the cell extends to infinity, where the electric field is indeed the applied one, at the electrodes, see Fig. 1. For $\langle i\rangle$ in Eq. (40) which is now the "average electric current in the absence of electric field $(\langle E\rangle=0)$, but in presence of the gravitational field," Ohshima uses an expression derived from his Eq. (4.14),

$$
\begin{aligned}
\langle i\rangle & =K_{1}\left(\langle E\rangle-E_{S E D}\right), \\
\langle i\rangle_{\langle E\rangle=0} & =-K_{1} E_{S E D} \quad \text { (Ohshima). }
\end{aligned}
$$

He then inserts Eqs. (43) and (44) into Eq. (40) and uses Eq. (42) to obtain his result,

$$
\mathbf{E}_{S E D}=\frac{-\Delta \rho \phi \mu_{\mathrm{E}}(1-\phi)}{K_{1}(1+\phi / 2)} \mathbf{g} \quad \text { (Ohshima). }
$$

Doing so, Ohshima uses a different definition of $\mu_{\mathrm{E}}$ and $\mathbf{E}_{S E D}$ than we do. In Ohshima's notation, we use

$$
\mathbf{v}_{p}=\mu_{\mathrm{E}}\langle\mathbf{E}\rangle \quad \text { (this article), }
$$

where $\langle\mathbf{E}\rangle$ is the average electric field in the absence of gravitational field in the colloidal suspension (which is in principle the measured-or often applied-electric field in the measurements). We have defined $\langle i\rangle_{\langle E\rangle=0}$ by

$$
\langle i\rangle_{\langle E\rangle=0}=\mu_{\mathrm{E}} \Delta \rho \phi g \quad \text { (this article). }
$$

What we define as the sedimentation potential follows simply from setting $\langle i\rangle=I=0$ in Eq. (7),

$$
\langle\mathbf{E}\rangle_{I=0}=\frac{-\Delta \rho \phi \mu_{\mathrm{E}}}{K} \mathbf{g} \text { (this article). }
$$

Using Eq. (47), we get

$$
E_{S E D}=\langle E\rangle_{I=0}=\frac{-\langle i\rangle_{\langle E\rangle=0}}{K} \quad \text { (this article). }
$$

Consequently,

$$
\begin{aligned}
\mu_{\mathrm{E}} & =\frac{1+\phi / 2}{1-\phi} \mu_{\mathrm{E}}^{\text {Ohshima }}, \\
E_{S E D} & =\frac{1+\phi / 2}{1-\phi} E_{S E D}^{\text {Ohshima }} .
\end{aligned}
$$

In principle, considering Eq. (50), one would think that there should be no difference between Eqs. (39) and (45). However, multiplying by $(1-\phi) /(1+\phi / 2)$, both sides of Eq. (39) would yield the same expression between $\mu_{\text {sedim }}^{\text {Ohshima }}$ and $E_{S E D}^{\text {Ohshima }}$ as we find between $\mu_{\text {sedim }}$ and $E_{S E D}$. The expression thus obtained would therefore be different from Eq. (45). The mistake made by Oshima arises when he inserts Eqs. (43) and (44) in the Onsager reciprocal relation Eq. (40). It is correct, as Ohshima does, to write

$$
\mu_{\mathrm{E}}=\left(\frac{\mathbf{v}_{p}}{\mathbf{E}}\right)_{g=0}\left(\frac{\mathbf{E}}{\langle\mathbf{E}\rangle}\right)_{g=0}=\frac{1+\phi / 2}{1-\phi} \mu_{\mathrm{E}}^{\text {Ohshima }} .
$$

However, it is not correct to write (this is what Ohshima does by inserting his Eq. (6.5) in his Eq. (8.4))

$$
\frac{1}{\Delta \rho \phi}\left(\frac{\langle\mathbf{i}\rangle}{\mathbf{g}}\right)_{\langle E\rangle=0}=\frac{1}{\Delta \rho \phi}\left(\frac{\langle\mathbf{i}\rangle}{\mathbf{E}_{S E D}}\right)_{\langle E\rangle=0}\left(\frac{\mathbf{E}_{S E D}}{\mathbf{g}}\right)_{\langle E\rangle=0}
$$

because it is contradictory to define the sedimentation electric field $\mathbf{E}_{S E D}$, i.e., the average electric field $\langle E\rangle$ created by the action of a gravitational field on charged particles when the electric field is zero $(\langle E\rangle=0)$. The sedimentation potential should be defined like we do, at zero electric current $(I=0)$. This can be best seen by comparing Eqs. (44) and (49),

$$
\begin{aligned}
& \frac{\left(E_{S E D}\right)_{\langle E\rangle=0}}{\langle i\rangle_{\langle E\rangle=0}}=\frac{\langle E\rangle_{\langle E\rangle=0}}{\langle i\rangle_{\langle E\rangle=0}}=\frac{-1}{K_{1}} \quad \text { (Ohshima), } \\
& \frac{\left(E_{S E D}\right)_{\langle i\rangle=0}}{\langle i\rangle_{\langle E\rangle=0}}=\frac{\langle E\rangle_{\langle i\rangle=0}}{\langle i\rangle_{\langle E\rangle=0}}=\frac{-1}{K} \quad \text { (this article). }
\end{aligned}
$$

It is clear that Ohshima's definition of the sedimentation electric field lacks consistency. Hence, Eq. (39) is correct whereas Eq. (45) is not. The sedimentation electric field for suspensions of any volume fraction, provided that the particles do not interact, is given by (we now use again the notations adopted in this article)

$$
\begin{aligned}
\mathbf{E}_{I=0} & =\frac{-\left(\bar{\rho}_{p}-\bar{\rho}_{w}\right) \phi \mu_{\mathrm{E}}(1-\phi \beta)}{K_{1}(1+2 \phi \beta)} \mathbf{g} \\
& \simeq \frac{-\left(\bar{\rho}_{p}-\bar{\rho}_{w}\right) \phi \mu_{\mathrm{E}}}{K_{1}} \mathbf{g} \text { for } \phi \ll 1 .
\end{aligned}
$$

This last relation is the one used by Ohshima et al. ${ }^{7}$ to test the Onsager relation for the relation found by solving the electroacoustic equations in the case of a dilute suspension of colloidal particles for all $\kappa a$ and zeta potentials. In that article, the authors use the definitions we use (written here in our notations): $E_{S E D}=E_{I=0}$ and $\mu_{\mathrm{E}}=\left(\mathbf{v}_{p} / \mathbf{E}\right)_{g=0}$.

\section{A small discussion about Ohshima's equation}

The core of the problem lies in Eq. (6.4) in Ref. 8, i.e., Eq. (44),

$$
\langle i\rangle=K_{1}\left(\langle E\rangle-E_{S E D}\right)
$$

This equation describes the relation between the macroscopic electric current $\langle i\rangle$ and the macroscopic electric field $\langle E\rangle$. The assumption made for this equation is, in Ohshima words, that there is "no applied electric field." This means that the existing macroscopic electric field $\langle E\rangle$ is determined by another condition. This condition is $\langle i\rangle=0$. Indeed, in a stationary process, with no charge consumption at the electrodes, the macroscopic current is equal to zero. With this condition, the macroscopic electric field is $\langle E\rangle_{\langle i\rangle=0}=E_{S E D}$. This last equation is therefore equivalent to Eq. (6.4)

If we want, in the general case, the macroscopic electric field in the suspension $\langle E\rangle$ to be equal to zero, we have to superpose a macroscopic electric field $\langle E\rangle_{\text {added }}$ of value $-E_{S E D}$. This added macroscopic electric field generates a current which is by definition $K \cdot\langle E\rangle_{\text {added }}$. The macroscopic electric current $\langle i\rangle$, in the general case, is thus the sum of the current with no imposed electric field $(=0)$ and of the current due to the added electric field ( $=K \cdot\langle E\rangle_{\text {added }}$ ); therefore, the sedimentation current, defined at no existing electric field, is

$$
\langle i\rangle_{\langle E\rangle=0}=-K \cdot E_{S E D}=-K \cdot\langle E\rangle_{\langle i\rangle=0} .
$$


Equation (6.4) in Ref. 8 should have been written, under the assumption that the existing electric field $\langle E\rangle$ is the sum of the electric field generated by the sedimentation $\langle E\rangle_{\langle i\rangle=0}$ associated to a current that is zero and an added macroscopic electric field $\left(\langle E\rangle-\langle E\rangle_{\langle i\rangle=0}\right)$ associated to a current $K\left(\langle E\rangle-\langle E\rangle_{\langle i\rangle=0}\right)$,

$$
\langle i\rangle=K\left(\langle E\rangle-\langle E\rangle_{\langle i\rangle=0}\right)=K\left(\langle E\rangle-E_{S E D}\right) .
$$

\section{B. Colloid Vibration Potential//ntensity 1. CVP}

In order to compare to what Ohshima and Dukhin did in Ref. 20, we define the colloid vibration potential by Eq. (20),

$$
\mathrm{CVP}=-(\mathbf{E})_{\mathbf{I}=\mathbf{0}}=\frac{\mu_{\mathrm{E}}}{K} \frac{\Delta \rho}{\rho} \phi \boldsymbol{\nabla} P_{I=0} .
$$

Note that strictly speaking, the CVP defined above is an electric field, not an electric potential. We note also that this equation is formally the same as Eq. (9) that was found in the case of SP. In the SP case, the pressure gradient originated from the gravitational field (and has no frequency dependence), whereas here the pressure is due to the acoustic wave. This is why SP can be seen as the static version of CVP. The electrophoretic mobility at no applied pressure is defined by

$$
\mu_{\mathrm{E}}=\left(\frac{\mathbf{v}_{p}}{\mathbf{E}}\right)_{\nabla P=\mathbf{0}} .
$$

In Ref. 15, the expression found by Ohshima et al. ${ }^{20}$ for CVP in concentrated suspensions was briefly commented. Their expression for CVP (Eq. (15) in their paper) reads

$$
\mathrm{CVP}=\frac{1-\phi}{1+\phi / 2} \frac{\mu_{\mathrm{E}}}{K_{1}} \frac{\Delta \rho}{\bar{\rho}_{w}} \phi \nabla P .
$$

The authors state that they have found this expression by analogy with Eq. (45), an equation they derived earlier, but also given in Ref. 8 and that we have discussed in Sec. III A. Since we have proven that Eq. (45) is not correct, it follows that Eq. (60) is also incorrect. The correct expression for CVP in (concentrated) suspensions of colloidal particles, using Eqs. (58) and (37), is

$$
\begin{aligned}
\mathrm{CVP} & =-(\mathbf{E})_{\mathbf{I}=\mathbf{0}}=\frac{1-\phi \beta}{1+2 \phi \beta} \frac{\mu_{\mathrm{E}}}{K_{1}} \frac{\bar{\rho}_{p}-\bar{\rho}_{w}}{\phi \bar{\rho}_{p}+(1-\phi) \bar{\rho}_{w}} \phi \boldsymbol{\nabla} P_{I=0} \\
& \simeq-(\mathbf{E})_{\mathbf{I}=\mathbf{0}}=\frac{\mu_{\mathrm{E}}}{K_{1}} \frac{\bar{\rho}_{p}-\bar{\rho}_{w}}{\bar{\rho}_{w}} \phi \boldsymbol{\nabla} P_{I=0} \quad \text { if } \phi \ll 1 .
\end{aligned}
$$

The last relation is the relation obtained by Enderby ${ }^{5}$ for low volume fractions by solving the electroacoustic equations.

\section{2. $\mathrm{CVI}$}

Dukhin et al. give a formulation for CVI in Ref. 18 where they also give an expression for the entropy production, their Eq. (6), which should be compared to our Eq. (12),

$$
\sigma=\frac{\mathbf{I} \cdot \mathbf{E}}{T}+\frac{1}{T} \boldsymbol{\nabla} P \cdot \mathbf{v}_{p}^{w} \quad \text { (Dukhin), }
$$

where $\mathbf{v}_{p}^{w}$ is the velocity of the particle in the reference frame of the solvent, as defined in Appendix A (the superscript $w$ stands for water). Note that for dimensions' sake we have changed their $\Delta P$ into $\nabla P$ and we have adapted notations of Dukhin et al. to ours. The corresponding forces-fluxes relations are

$$
\begin{aligned}
\mathbf{v}_{p}^{w} & =d_{P P}^{*} \boldsymbol{\nabla} P+d_{P E}^{*} \mathbf{E} \text { (Dukhin), } \\
\mathbf{I} & =d_{E P}^{*} \boldsymbol{\nabla} P+d_{E E}^{*} \mathbf{E} \text {. }
\end{aligned}
$$

Dukhin et al. then give what they call the Onsager relation,

$$
\frac{\mathbf{v}_{p}^{w}}{(\mathbf{I})_{\nabla P=0}}=\frac{\mathbf{E}}{(\boldsymbol{\nabla} P)_{\mathbf{I}=\mathbf{0}}} \quad \text { (Dukhin). }
$$

They refer to the book edited by Kruyt for this expression. ${ }^{21}$ However, the Onsager relation given in Ref. 21 was given (p. 206, Eq. (24)) in the context of streaming potential and electroosmosis and reads

$$
\frac{\text { vol }}{(\mathbf{I})_{\nabla P=0}}=\frac{\mathbf{E}}{(\boldsymbol{\nabla} P)_{\mathbf{I}=\mathbf{0}}}=\frac{\varepsilon_{1} \varepsilon_{0} \zeta}{\eta} \frac{1}{K_{1}} .
$$

The term "vol" appearing in Eq. (65) in place of $\mathbf{v}_{p}^{w}$ referred to the electroosmotic flow velocity multiplied by the area of the cross section of the capillary. This term "vol" is therefore similar to a volume flux, quite different from the particle's velocity $\mathbf{v}_{p}^{w}$ given in Eq. (64). For more information about the Onsager relation in the case of electroosmosis/streaming potential, we refer to the work of Mazur and Overbeek in Refs. 22 and 23. From Eq. (63), it is also clear that the Onsager relation to be satisfied, in the case of Dukhin et al., should be

$$
\left(\frac{\mathbf{v}_{p}^{w}}{\mathbf{E}}\right)_{\nabla P=0}=\left(\frac{\mathbf{I}}{\nabla P}\right)_{E=0} \text { (Dukhin). }
$$

Dukhin et al. then derive relations for the particle's electrophoretic mobility and the electric current and claim that their found relations satisfy their Eq. (40), which is quite similar to our Eq. (66). Equation (66) should be compared to Eq. (17) given in Sec. II. It is clear from the differences between the equations that there is an inconsistency in Eq. (66): when the particle and the solvent have the same density, Eq. (17) gives that there is no CVI signal, whereas there is a CVI signal in the case defined by Eq. (66).

In Refs. 9 and 10, Dukhin et al. detail their derivation further and give relations between CVP/CVI and expressions for the CVI at low frequencies. They define as "CVP $\omega \rightarrow 0$ " the electric field $\mathbf{E}$ in Eq. (64). They state "Much less is known about its [= the Onsager reciprocity relationship] validity in the case of alternating fields." We note that recently ${ }^{15}$ it was demonstrated that the Onsager reciprocity relationship is indeed valid for alternating fields. Dukhin et al. define the particle mobility by $\mu=\left(\mathbf{v}_{p}^{w} / \mathbf{E}\right)_{\nabla P=0}$ and the CVI by CVI $\mathrm{I}_{\omega \rightarrow 0}$ $=(\mathbf{I})_{\nabla E=0}$ and find that, see Eqs. (43) and (44) p. 503 in Ref. 10 and the text above Eq. (43) p. 503 in Ref. 10 (or alternatively Eqs. (5.14) and (5.15) in Ref. 9),

$$
\begin{aligned}
\mathrm{CVP}_{\omega \rightarrow 0} & =\frac{\mathrm{CVI}_{\omega \rightarrow 0}}{K} \text { (Dukhin), } \\
\mu & =\frac{-\varepsilon_{1} \varepsilon_{0} \zeta}{\eta} \frac{K}{K_{1}},
\end{aligned}
$$

where $\varepsilon_{1} \varepsilon_{0}$ is the dielectric permittivity of the solvent (water) and $\eta$ its viscosity. The zeta potential of the particle is given by $\zeta$. The expression given for $\mu$ comes from modifying the 
expression for electroosmosis given in Eq. (65) as in Eq. (64) and using the relation between CVP and CVI given in the first line of Eq. (67). Dukhin et al. state that Eq. (67) is valid for the case originally considered by Smoluchowski, i.e., small double layers and negligible surface conductivity. The conductivity $K_{1}$ is the conductivity of the electrolyte and $K$ of the suspension for $\omega=0$. The ratio $K / K_{1}$ is given in Eq. (37) and is given by Dukhin et al. in their Eq. (45) p. 503 in Ref. 10 for the case that the zeta potentials are low and $\beta \simeq-1 / 2$. We note that in case that the pressure gradient is the same in the CVP/CVI experiment, we find, like Dukhin et al. do, the first line of Eq. (67) but with a minus sign difference (see Eqs. (17) and (20) given above, from which it becomes clear why there should be a minus sign). Dukhin et al. find for the CVI signal,

$$
\mathrm{CVI}_{\omega \rightarrow 0}=\frac{\varepsilon_{1} \varepsilon_{0} \zeta}{\eta} \frac{K}{K_{1}} \frac{\bar{\rho}_{p}-\rho}{\rho} \phi \nabla P^{*} \quad \text { (Dukhin), }
$$

where they define the "effective pressure gradient" by $\nabla P_{\omega \rightarrow 0}$ and $\nabla P^{*}$ by the "pressure gradient by the sound wave." The two are related by their Eq. (42) p. 503 in Ref. 10,

$$
\boldsymbol{\nabla} P_{\omega \rightarrow 0}=\frac{\bar{\rho}_{p}-\bar{\rho}_{w}}{\bar{\rho}_{w}} \phi \nabla P^{*} \quad \text { (Dukhin). }
$$

The inconsistency in Eq. (66) was solved in Refs. 10 and 9 by the authors by introducing the "effective pressure gradient" which ensures that there is indeed no CVI signal in the case that the particle's density matches the density of the solvent. The discrepancy found by Dukhin comparing the expressions for CVI (Eq. (68) corresponding to Eq. (46) p. 503 in Ref. 10) and the expression found for ESA by O'Brien (Eq. (47) p. 504 in Ref. 10) corresponds to the difference between our Eqs. (18) and (19). Dukhin assumes [p. 506 in Ref. 10] that the nullity of the CVI when the colloid and the suspending medium have the same absolute density implies that the density contrast term should be $\left(\bar{\rho}_{p}-\rho\right)$, like in Eq. (68). However, if the suspending medium and the colloidal particle have the same density, it follows that the solvent and the colloidal particle have also the same density, so that the density contrast is also proportional to $\left(\bar{\rho}_{p}-\bar{\rho}_{w}\right)$. This is not contradictory, as we have shown in the text between Eqs. (18) and (19). The difference between the CVI and the ESA expressions is in fact due to the different electrophoretic mobilities appearing in these equations and originates from the conditions on the volume and mass fluxes in the different theoretical and experimental conditions. The ESA and CVI/CVP principles are shown in Fig. 2.

\section{ESA}

Instead of applying an acoustic wave on the suspension, which leads to the generation of electric potential differences (CVP) or electric currents (CVI), it is possible to apply an electric field, which in turn will generate an acoustic signal known as the ESA. We refer, for more details, to Refs. 24 and 6 and to Ref. 15 where ESA was already discussed in the frame of Onsager's relation. ESA differs from CVP/CVI in the sense that the acoustic wave is generated at the electrodes, as discussed by O'Brien in Ref. 24. Indeed, when an electric field is applied on the solution, it creates a volume flow. This flow is stopped by the electrode, creating a pressure wave, like in the "water hammer" phenomenon. In the "water hammer" phenomenon, the water before the wall is moving towards the wall until a pressure surge, created by the presence of the wall, stops it. The relation between water velocity and the pressure surge was found by Joukowski ${ }^{26}$ and may be deduced easily from the definition of acoustical impedance,

$$
P_{0}=\frac{z_{S} z_{B}}{z_{S}+z_{B}} v^{l a b}
$$

in which $P_{0}$ is the pressure surge, $z_{S}$ is the acoustical impedance of the solution, $z_{B}$ the one of the boundary, and $v^{\text {lab }}$ the velocity of water. If the boundary, here the electrode, is hard, then $z_{S} z_{B} /\left(z_{S}+z_{B}\right)$ reduces to $z_{S}$, which means that only the water bears the compression. In electroacoustic of solutions and suspensions, volume flow and mass flow are not proportional, contrary to a system made of pure water. The boundary condition of "no flow through" means no volume flow $\left(\mathbf{v}_{v o l}^{l a b}=0\right)$ at the electrode. It implies that, seen from a distance, there is a mass flow through the electrode. In reality, there is no mass flow strictly through the electrode, but there
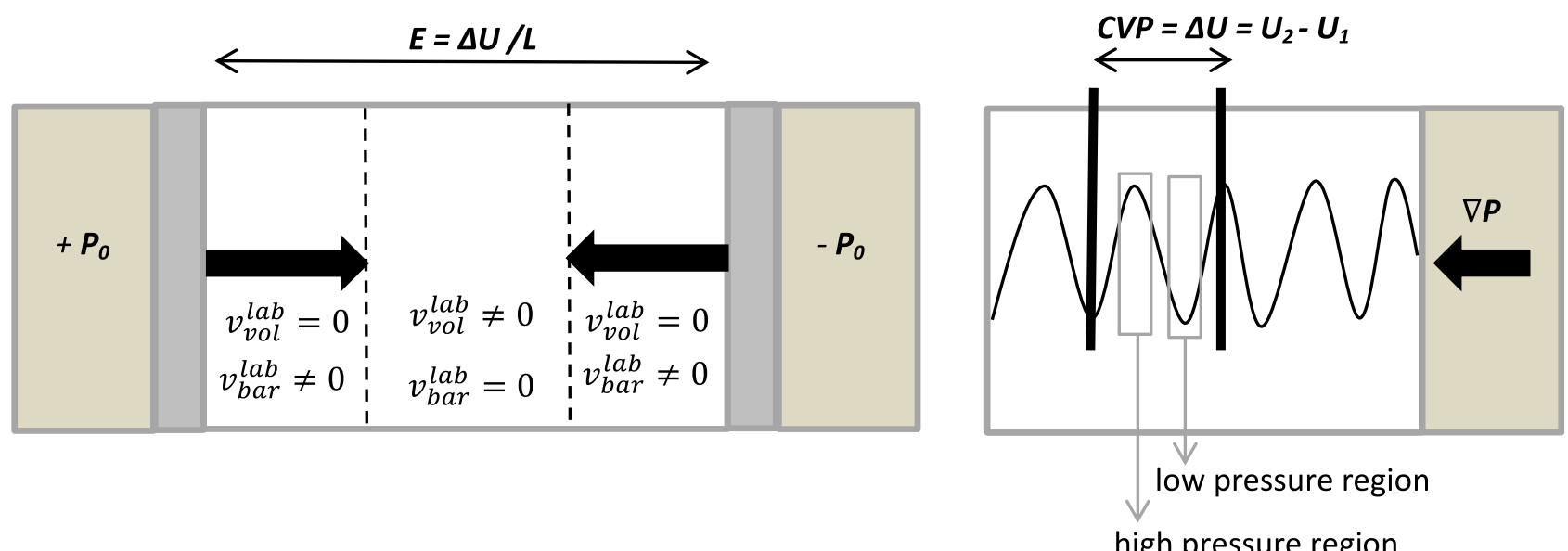

FIG. 2. Schematic views of the working principles for ESA (left) and CVP/CVI (right). For ESA, an electric field E is applied between two electrodes separated by a distance L. The pressure wave generated at the electrode is measured as the amplitude $P_{0}$. In the region affected by the pressure wave, the volume flux $v_{v o l}^{\text {lab }}$ is zero. For CVP/CVI, an acoustic wave $\nabla P$ is applied, generating an electric potential difference CVP at any odd multiple of half the wavelength. The corresponding electric field can be evaluated by integrating CVP over half a wavelength. 


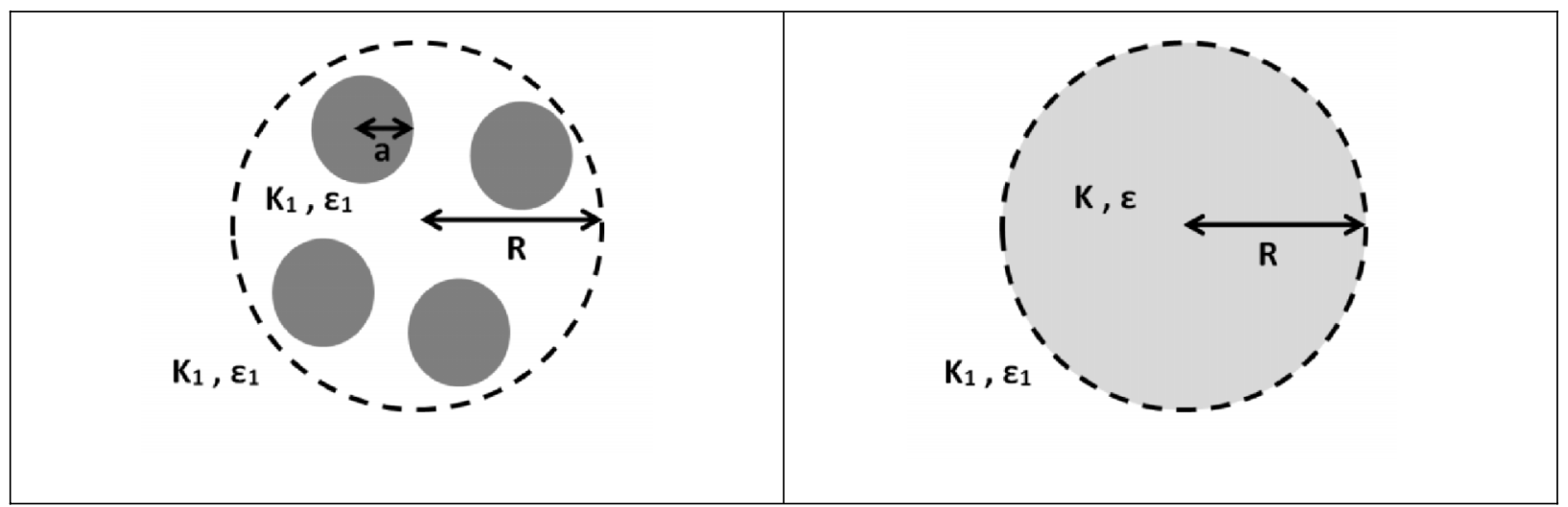

FIG. 3. Left: sphere $\mathrm{A}$ of radius $\mathrm{R}$ is defined so as to contain $\mathrm{N}$ particles of radius a and dipole coefficient $\beta$. The conductive and dielectric properties of sphere $\mathrm{A}$ and the electrolyte are $(K, \varepsilon)$ and $\left(K_{1}, \varepsilon_{1}\right)$. Right: sphere $\mathrm{B}$ of radius $\mathrm{R}$ is defined so as to contain a fluid that has the same conductive and dielectric properties $(K, \varepsilon)$ as sphere $\mathrm{A}$ and is immersed in the same electrolyte of properties $\left(K_{1}, \varepsilon_{1}\right)$.

may be an accumulation or a depletion in heavy particles near the electrode, yielding the same boundary condition.

The ESA problem thus reduces to find the volume flow due to the application of the electric field when no pressure is applied, $\left(\mathbf{v}_{v o l}^{l a b}\right)_{\mathbf{V P}=\mathbf{0}}$. A Chasles-like relation, analysed in Ref. 16, yields a link between velocities of several referentials,

$$
\mathbf{v}_{v o l}^{l a b}=\mathbf{v}_{\text {vol }}^{b a r}+\mathbf{v}_{b a r}^{l a b} \text {. }
$$

In the case of ESA, it is easy to estimate $\mathbf{v}_{l a b}^{b a r}$. At the onset of the experiment, no forces are applied to the system, which is then are rest, and $\mathbf{v}_{b a r}^{l a b}(t=0)=0$. When an electric field is applied to the system, electroneutrality insures that no net electric force is applied to the system and therefore $d \mathbf{v}_{\text {bar }}^{l a b} / d t$ $=0$ from which follows that $-\mathbf{v}_{l a b}^{b a r}=\mathbf{v}_{b a r}^{l a b}=\mathbf{v}_{b a r}^{l a b}(t=0)=0$.

Moreover,

$$
\mathbf{v}_{v o l}^{b a r}=-\mathbf{v}_{b a r}^{v o l}=-\frac{\mathbf{J}^{v o l}}{\rho} .
$$

Combining the three previous equations, we get

$$
\begin{aligned}
P_{0} & =\frac{z_{S} z_{B}}{z_{S}+z_{B}}\left(\mathbf{v}_{v o l}^{l a b}\right)_{\mathbf{\nabla}=\mathbf{0}} \\
& =-\frac{z_{S} z_{B}}{z_{S}+z_{B}}\left(\frac{\mathbf{J}^{v o l}}{\rho}\right)_{\mathbf{\nabla} \mathbf{P}=\mathbf{0} .}
\end{aligned}
$$

Consequently, an Onsager-like relationship may be written for CVP and ESA, if we define ESA as ESA $=\left(P_{0} / E\right)_{\nabla \mathbf{P}=\mathbf{0}}$ (i.e., the pressure surge at the electrode per unit of applied electric field without applying an acoustic field to the bulk) and combine it with an Onsager relation for CVI,

$$
\begin{aligned}
\mathrm{ESA} & =-\frac{z_{S} z_{B}}{z_{S}+z_{B}}\left(\frac{\mathbf{J}^{\mathrm{vol}}}{\rho} \frac{1}{\mathbf{E}}\right)_{\mathbf{\nabla P}=\mathbf{0}} \\
& =-\frac{z_{S} z_{B}}{z_{S}+z_{B}}\left(\frac{I}{\boldsymbol{\nabla} P / \rho}\right)_{\mathbf{E}=\mathbf{0}} \\
& =\frac{z_{S} z_{B}}{z_{S}+z_{B}} K \cdot \mathrm{CVI} .
\end{aligned}
$$

The conductivity $K$ can be obtained as function of the volume fraction from Eq. (37). Using an Onsager relation for the CVP, we get

$$
\mathrm{ESA}=-\frac{z_{S} z_{B}}{z_{S}+z_{B}} \mu_{\mathrm{E}} \frac{\Delta \rho}{\rho} \phi,
$$

in which $\Delta \rho / \rho$ means "the absolute density of the colloid minus the absolute density of the solution divided by the absolute density of the suspension."

O'Brien wrote in $2003^{6}$ the same equation, with slightly different notations. Converted into our notation system, this relation is (see (1.4) in Ref. 6)

$$
\mathrm{ESA}=\frac{z_{S} z_{B}}{z_{S}+z_{B}} \phi \mu_{\mathrm{E}}^{*} \frac{\Delta \rho}{\bar{\rho}_{w}} .
$$

The sign is only a convention: the ESA effect at one electrode is equal to minus the ESA effect at the other electrode. The electrophoretic mobility $\mu_{\mathrm{E}}^{*}$ is defined in the barycentric referential, and as shown in Appendix A, it is linked to the electrophoretic mobility in the referential of volume by $\mu_{\mathrm{E}}^{*}=\mu_{\mathrm{E}}\left(\bar{\rho}_{w} / \rho\right)$.

\section{CONCLUSION}

In this article, we have clarified the relations existing for SP, SI, CVP, CVI, and ESA as function of the electrophoretic mobility of a colloidal particle. Depending on the experiment, caution should be taken in the use of the appropriate referentials to relate these signals to the electrophoretic mobilities. As we have shown, all quantities of interest can be expressed in the referential of zero volume flux, which is equivalent to the laboratory frame of reference for SP/SI, but they are often expressed in other referentials. This implies that the electrophoretic mobility should be converted, according to the derivations shown in Appendix A, into the desired electrophoretic mobility. The desired electrophoretic mobility is often the electrophoretic mobility $\mu_{\mathrm{E}}$ that is measured by electrophoresis (where a condition of zero volume flux is applied). This mobility $\mu_{\mathrm{E}}$ is also the one appearing in most equations defining the CVP, see Eq. (58). The mobility derived from theoretical/numerical considerations, in the case where no condition is applied, is $\mu_{0}$ according to our notations. The mobility defined as $\mu_{\mathrm{E}}^{*}$, when a zero mass flow condition is applied, is the mobility usually used in ESA, see Eq. (75). In 
general, all electroacoustic signals are proportional to

$$
\begin{aligned}
(1-\phi) \mu_{0} \frac{\bar{\rho}_{p}-\bar{\rho}_{w}}{\rho} & =\mu_{0} \frac{\bar{\rho}_{p}-\rho}{\rho}=\mu_{\mathrm{E}} \frac{\bar{\rho}_{p}-\bar{\rho}_{w}}{\rho} \\
& =\mu_{\mathrm{E}}^{*} \frac{\bar{\rho}_{p}-\bar{\rho}_{w}}{\bar{\rho}_{w}} .
\end{aligned}
$$

\section{ACKNOWLEDGMENTS}

The authors would like to warmly thank Dick Bedeaux, Olivier Bernard, and Marie Jardat for fruitful discussions. Financial support of the Agence Nationale de la Recherche in the frame of the project Celadyct (No. ANR-12-BS080017-01) is acknowledged. The authors also gratefully thank the University Pierre et Marie Curie (UPMC) for granting C. Chassagne a temporary position as invited professor. Parts of this article were written during the stay of C. Chassagne at the UPMC.

\section{APPENDIX A: RELATIONS BETWEEN MOBILITIES}

We have in Secs. II-IV expressed the SP, CVP/CVI, and ESA responses in terms of the electrophoretic mobilities $\mu_{\mathrm{E}}^{\text {lab, vol. flux. }=0}$ (SP, CVP/CVI conditions) and $\mu_{\mathrm{E}}^{\text {lab, mass. flux }=0}$ (ESA conditions). The aim of this appendix is to link these mobilities to the electrophoretic mobility measured by electrophoresis. To define the electrophoretic mobility in this case, we will follow the procedure adopted by O'Brien and coworkers. ${ }^{25}$ We consider a colloidal particle travelling at a velocity $\mathbf{v}_{p}^{l a b}$ or a set of colloidal particles travelling at the same velocity $\mathbf{v}_{p}^{l a b}$, in a solvent of velocity $\mathbf{v}_{w}^{l a b}$ under the action of an applied electric field $\mathbf{E}$. The velocity of the particle can be written in the reference frame of the solvent as

$$
\mathbf{v}_{p}^{w}=\mathbf{v}_{p}^{l a b}-\mathbf{v}_{w}^{l a b} .
$$

The evaluation of $\mathbf{v}_{p}^{w}$ can be splitted into two sub-problems.

Sub-problem 1: the applied electric field is set equal to zero, i.e., $\mathbf{E}=\mathbf{0}$, which force $\mathbf{F}_{1}$ is required to ensure that the particles have a velocity $\mathbf{v}_{p}^{w}$ ? The force $\mathbf{F}_{1}$ is then proportional to $\mathbf{v}_{p}^{w}$ and independent of $\mathbf{E}$. We define $\mathbf{F}_{1}=a_{1} \mathbf{v}_{p}^{w}$.

Sub-problem 2: the applied electric field is $\mathbf{E}$, which force $\mathbf{F}_{2}$ is required to ensure that the particles have a velocity $\mathbf{v}_{p}^{w}$ $=0$ ? The force $\mathbf{F}_{2}$ is then proportional to $\mathbf{E}$ and independent of $\mathbf{v}_{p}^{w}$. We define $\mathbf{F}_{2}=a_{2} \mathbf{E}$.

Because of the linearity of the equations and the fact that no net force is applied to the colloidal particle in the total problem (= both the particle and the solvant are moving in an applied electric field), we get $\mathbf{F}_{1}+\mathbf{F}_{2}=0$ and we find $\mathbf{v}_{p}^{w}=-a_{2} / a_{1} \mathbf{E}$. The coefficients of proportionality $a_{1}$ and $a_{2}$ do not depend on any assumptions about volume or mass fluxes; hence, we can define $\mathbf{v}_{p}^{w}=\mu_{0} \mathbf{E}$, where $\mu_{0}$ is the electrophoretic mobility of the colloidal particle that is usually derived in electrokinetic theories. The experimental conditions for determining the electrophoretic mobility $\mu_{\mathrm{E}}$ are usual such that $\mathbf{v}_{w}^{l a b} \simeq 0$ and therefore, for experiments, $\mathbf{v}_{p}^{w}=\mathbf{v}_{p}^{l a b}=\mu_{\mathrm{E}} \mathbf{E}$. Most experimental setups are done in condition of zero total volume flow, in closed cells. Therefore, $\mu_{\mathrm{E}}=\mu_{\mathrm{E}}^{l a b \text {, vol. flux }=0}$. We can now express the mobilities found in the case of CVP and
ESA as function of $\mu_{0}$,

$$
\begin{aligned}
\mu_{\mathrm{E}}^{l a b, \text { vol. flux }=0} & =\left(\frac{\mathbf{v}_{p}^{l a b}}{\mathbf{v}_{p}^{w}} \frac{\mathbf{v}_{p}^{w}}{\mathbf{E}}\right)^{l a b, \text { vol. flux }=0} \\
& =\mu_{0}\left(\frac{\mathbf{v}_{p}^{l a b}}{\mathbf{v}_{p}^{w}}\right)^{l a b, \text { vol. flux }=0}, \\
\mu_{\mathrm{E}}^{l a b, \text { mass flux }=0} & =\left(\frac{\mathbf{v}_{p}^{l a b}}{\mathbf{v}_{p}^{w}} \frac{\mathbf{v}_{p}^{w}}{\mathbf{E}}\right)^{l a b, \text { mass. flux }=0} \\
& =\mu_{0}\left(\frac{\mathbf{v}_{p}^{l a b}}{\mathbf{v}_{p}^{w}}\right)^{l a b, \text { mass flux }=0}
\end{aligned}
$$

In the case of zero volume flux (see Eq. (4) for a definition of the volume flux),

$$
\mathbf{v}_{w}^{l a b}=\frac{-\phi}{(1-\phi)} \mathbf{v}_{p}^{l a b} .
$$

Therefore, from Eq. (A1),

$$
\mathbf{v}_{p}^{w}=\frac{1}{(1-\phi)} \mathbf{v}_{p}^{l a b} .
$$

We finally get

$$
\mu_{\mathrm{E}}^{l a b, \text { vol. flux }=0}=(1-\phi) \mu_{0} .
$$

In the case of zero mass flux, see Eq. (3),

$$
\mathbf{v}_{w}^{l a b}=\frac{-\phi \bar{\rho}_{p}}{(1-\phi) \bar{\rho}_{w}} \mathbf{v}_{p}^{l a b} .
$$

Therefore, from Eq. (A1),

$$
\mathbf{v}_{p}^{w}=\frac{(1-\phi) \bar{\rho}_{w}+\phi \bar{\rho}_{p}}{(1-\phi) \bar{\rho}_{w}} \mathbf{v}_{p}^{l a b}=\frac{\rho}{(1-\phi) \bar{\rho}_{w}} \mathbf{v}_{p}^{l a b} .
$$

We finally get

$$
\mu_{\mathrm{E}}^{\text {lab,mass flux }=0}=(1-\phi) \mu_{0} \frac{\bar{\rho}_{w}}{\rho} .
$$

It follows from Eqs. (A5) and (A8) and the fact that the electrophoretic mobility $\mu_{\mathrm{E}}$ measured during standard electrophoresis experiments is given by $\mu_{\mathrm{E}}=\mu_{\mathrm{E}}^{\text {lab, vol. flux }=0}$ that

$$
\mu_{\mathrm{E}}^{\text {lab,mass flux }=0}=\frac{\bar{\rho}_{w}}{\rho} \mu_{\mathrm{E}} .
$$

This result holds at any volume fraction of colloids.

\section{APPENDIX B: MAXWELL GARNETT EQUATION}

We recall here the derivation to obtain the conductivity of a system of dielectric colloidal particles suspended in an electrolyte for an arbitrary volume fraction $\phi$ of the colloid. This derivation can also be found in p. 279 in Ref. 10. Let us consider two macroscopic spheres of same radius $R$. Sphere $A$ consists of an electrolyte solution containing $N$ colloidal particles of radius $a$ and dipole coefficient $\beta$ and sphere $B$ consists of an homogeneous fluid of dipole coefficient $\beta_{B}$. An illustration is given in Fig. 3. We choose this fluid so as to have the same dielectric and conductive properties as sphere $A$. Both spheres are immersed in the same electrolyte solution that is also the same electrolyte as contained in sphere $A$. 
When the same electric field $\mathbf{E}$ is applied to the spheres, the electric potentials created outside $A$ and $B$ are

$$
\begin{aligned}
& \Psi_{A}=-\mathbf{E} \cdot \mathbf{r}+N \beta a^{3} \frac{\mathbf{E} \cdot \mathbf{r}}{r^{3}}, \\
& \Psi_{B}=-\mathbf{E} \cdot \mathbf{r}+\beta_{B} R^{3} \frac{\mathbf{E} \cdot \mathbf{r}}{r^{3}} .
\end{aligned}
$$

The dipole coefficient can be evaluated from solving the Laplace equation $\nabla^{2} \Psi_{B}=0$ together with the necessary boundary conditions stemming from Maxwell's relations (continuity of the electric potential and the normal component of the displacement field). One gets

$$
\beta_{B}=\frac{\widetilde{K}-\widetilde{K}_{1}}{\widetilde{K}+2 \widetilde{K}_{1}},
$$

where we used that the complex conductivity can be written in the general case: $\widetilde{K}=K+i \omega \varepsilon_{0} \varepsilon$, where $K$ is the DC conductivity and $\varepsilon_{0} \varepsilon$ the dielectric permittivity of the considered medium. By definition, we have $\Psi_{A}=\Psi_{B}$, and from Eq. (B1), it then follows

$$
\widetilde{K}=\widetilde{K}_{1} \frac{1+2 \phi \beta}{1-\phi \beta} \text { with } \phi=N a^{3} / R^{3} .
$$

When the volume fraction of the colloidal particles is low, one gets the relation

$$
\widetilde{K} \simeq \widetilde{K}_{1}(1+3 \phi \beta)
$$

${ }^{1}$ A. J. Rutgers, Physica 5, 54 (1938) [Nature 157, 74-76 (1946) (in English)].

${ }^{2}$ B. J. Marlow, D. Fairhurst, and H. P. Pendse, Langmuir 4, 611-626 (1988).

${ }^{3}$ R. W. O'Brien, J. Fluid Mech. 190, 71-86 (1988).
${ }^{4}$ A. Dukhin and P. Goetz, U.S. patent 6109098 A (30 June 1998).

${ }^{5}$ J. A. Enderby, Proc. R. Soc. A 207, 329 (1951).

${ }^{6}$ R. W. O'Brien, A. Jones, and W. N. Rowlands, Colloids Surf., A 218, 89-101 (2003).

${ }^{7}$ H. Ohshima, T. W. Healy, and L. R. White, J. Chem. Soc., Faraday Trans. 2 80, 1299-1317 (1984).

${ }^{8}$ H. Ohshima, Adv. Colloid Interface Sci. 88, 1-18 (2000).

${ }^{9}$ A. S. Dukhin and J. P. Goetz, Characterization of Liquids, Nano- and Microparticulates, and Porous Bodies Using Ultrasound (Elsevier, 2010).

${ }^{10}$ Interfacial Electrokinetics and Electrophoresis, edited by A. Delgado, Surfactant Science Series Vol. 106 (Marcel Dekker, 2002).

${ }^{11}$ M. Ozaki, T. Ando, and K. Mizuno, Colloids Surf., A 159, 477-480 (1999).

${ }^{12}$ S. Uddin, M. Mirnezami, and J. A. Finch, Physicochem. Engin. Asp. 371, 65-70 (2010).

${ }^{13}$ F. Booth, J. Chem. Phys. 22, 1956-1968 (1954).

${ }^{14}$ S. R. de Groot, P. Mazur, and J. T. G. Overbeek, J. Chem. Phys. 20, 1825 (1952).

${ }^{15}$ C. Chassagne and D. Bedeaux, J. Chem. Phys. 141, 044703 (2014).

${ }^{16} \mathrm{~S}$. Gourdin-Bertin, C. Chassagne, O. Bernard, and M. Jardat, "Onsager's reciprocal relations in electrolyte solutions Part I: Sedimentation and electroacoustics," J. Chem. Phys. (submitted).

${ }^{17}$ J. F. Dufreche, M. Jardat, T. Olynyk, O. Bernard, and P. Turq, J. Chem. Phys. 117, 3804 (2002).

${ }^{18}$ A. S. Dukhin, V. Shilov, and Y. Borkovskaya, Langmuir 15(10), 3452 (1999).

${ }^{19}$ J. F. Richardson and W. N. Zaki, Chem. Engineer. Sci. 3, 65-73 (1954).

${ }^{20}$ H. Ohshima and A. S. Dukhin, J. Colloid Interface Sci. 212, 449 (1999).

${ }^{21}$ H. R. Kruyt, Colloid Science, Irreversible Systems Vol. 1 (Elsevier, New York, 1952).

${ }^{22}$ S. R. de Groot and P. Mazur, Non-Equilibrium Thermodynamics (Dover Publications, New York, 1984).

${ }^{23}$ P. Mazur and J. T. G. Overbeek, Recl. Trav. Chim. Pays-Bas 70, 83 (1951).

${ }^{24}$ R. W. O' Brien, D. W. Cannon, and W. N. Rowlands, J. Colloid Interface Sci. 173, 406 (1995).

${ }^{25}$ R. W. O' Brien and L. R. White, J. Chem. Soc., Faraday Trans. 2 74, $1607-$ 1626 (1978).

${ }^{26}$ N. Joukowski, Ann. Ponts Chaussees 8(25), 199-221 (1907), (translated by M. Goupil), see http://gallica.bnf.fr/ark:/12148/bpt6k612588b/f200.image. 